\title{
Beobachtungen
}

\section{bei der isoelektrischen Trennung von menschlichem Transferrin $\left.{ }^{1}\right)$}

\author{
.Von H. E. Reis, O. Wetter und R. Pfeiffer unter technischer Mitarbeit von S. Beforth \\ Aus der Inneren Klinik und Poliklinik (Tumorforscbung) der Rubruniversität, Klinikum Essen \\ (Direktor: Prof. Dr. C. G. Schmidt)
}

(Eingegangen am 24. November 1969)

Eisenfreies Transferrin konnte isoelektrisch in 2 Komponenten getrennt werden, deren isoelektrischer Punkt bei pH 5,8 und 5,4 lag. Die Absättigung des Transferrins mit Eisen änderte das isoelektrische Muster prinzipiell nicht. Der isoelektrische Punkt beider Komponenten wurde durch die Eisenbindung um $0,3 \mathrm{pH}$-Einheiten erniedrigt. Durch die Untersuchung der isolierten Komponenten in der Stärkegelelektrophorese ließ sich dieser Effekt ebenfalls nachweisen und zeigen, daß es sich wahrscheinlich nicht um genctische Varianten handelt.

Weiterhin wurde beobachtet, daß die Verbringung eisenbeladenen Transferrins in den Ampholyten (Polyaminocarboxylsäuren) einen Teil des Eisens aus dem Transferrin-Eisen-Komplex freisetzt. Sekundär kommt es dann zur Bildung eines Eisen-Ampholyt-Komplexes, der sich durch Gelchromatographie abtrennen läßt. Diescr Effekt ist bei Studien der Bindungskapazität mit isoelektrischen Verfahren zu berïcksichtigen.

\section{Observations on the isoelectric separation of human transferrin}

Iron-free transferrin was separated isoelectrically into 2 components with isoelectric points at $\mathrm{pH} 5.8$ and 5.4. After saturation of the transferrin with iron, the isoelectric pattern remained essentially unchanged. Combination with iron lowered the isoelectric point of each component by pH 0.3 to 5.5 and 5.1 respectively. This effect has also been observed with the isolated components in starch gel electrophoresis. The two components observed by starch gel electrophoresis do not represent genetic variants.

Further it was found that by transfer into the ampholyte part of the iron dissociates from the transferrin-iron complex. A secondary reaction then leads to the formation of an iron-ampholyte complex, which can be separated by gel chromatography. This effect has to be taken into account in studies on binding capacity with isoelectric methods.

Die strukturellen Voraussetzungen der in der Stärkegelelektrophorese beobachteten Heterogenität menschlichen Transferrins sind bisher nicht bekannt. $\mathrm{Da}$ als $\mathrm{N}$-terminale Aminosäure nur Valin bestimmbar ist und die Elektrophorese in dissoziierenden Medien keinen Anhalt für eine mehrkettige Struktur liefert, scheidet eine quaternäre Allomerie nach Art der Hämoglobin-Typen als Ursache der Varianten innerhalb des Transferrin-Systems aus. Nachdem im Plasma und in verschiedenen Körperflüssigkeiten (1-4) Transferrine mit unterschiedlichem Sialinsäuregehalt gefunden wurden, wird als Ursache der Variabilität in zunehmendem Maße eine prosthetische Allomerie diskutiert. Eine derartige; sich nur auf den Kohlenhydratanteil des Moleküls erstreckende genetische Variation wird von JEPPSON und SJöQUisT (5) zur Erklärung unterschiedlicher chromatographischer Eigenschaften der von ihm beobachteten 2 Hauptkomponenten menschlichen Transferrins herangezogen.

Ziel der nachfolgend beschriebenen Untersuchungen war es, mit einer isoelektrischen Methode zu prüfen, ob sich eine Heterogenität von Transferrin feststellen läßt. Weiterhin sollte untersucht werden, ob sich unter den Bedingungen der Eisenbelastung das isoelektrische Verhalten ändert.

\section{Material und Methodik}

Eisenfreies Transferrin (Charge Nr. TEE 04) wurde von den Behringwerken Marburg/Lahn bezogen. $\mathrm{FeCl}_{3} \cdot 6 \mathrm{H}_{2} \mathrm{O}$ der $\mathrm{Fa}$. Merck und ${ }^{50} \mathrm{Fe}$ als Chlorid in $\mathrm{HCl}-\mathrm{Lösung}$ der $\mathrm{Fa}$. Hoechst (spez.

1) Mit Unterstützung der Deutschen Forschungsgemeinschaft.
Aktivität $>10 \mathrm{mC} / \mathrm{mg}$ ) wurden verwandt. Trägerampholyte (synthetische Polyaminocarboxylsäuren M. G. 500-700) der pHBereiche 3-10 und 5-7 bezogen wir von der Fa. LKB-Producter $A B$, Bromma Stockholm/Schweden.

Bei der Durchführung der isoelektrischen Trennung folgten wir im wesentlichen der von VeSTERBERG und SvensSON (6) beschriebenen Methode in der von uns bereits früher geschilderten Art (7). Eine Übersicht über Entwicklung, Prinzip, Gerät und Ergebnisse der isoelektrischen Trennung ist an anderer Stelle mitgeteilt (7a). Dazu wurde das ebenfalls von der Fa. LKB-Producter AB, Bromma Stockholm/Schweden angebotene Gerät, eine Säule des Typs LKB 8101, ein Gradientenmischer Typ 8121, eine Spannungsquelle Typ $3371 \mathrm{C}$ und ein Fraktionssammler Ultrarac 7000 benutzt. In der Säule des Typs LKB 8101 wurde über den Gradientenmischer LKB 8121 ein Saccharosedichtegradient aus einer spezifisch leichteren Lösung (Ampholyt ohne Saccharose) und einer spezifisch schwereren Lösung (Ampholyt plus Saccharose) aufgebaut. Pro Versuch wurden maximal $20 \mathrm{mg}$ Protein eingesetzt. Die Probe wurde bei der Vorbereitung der spezifisch leichteren Lösung zugesetzt. Die Polung erfolgte so, da $\beta$ sich die Anode (Anodenlösung mit Schwefel- oder Orthophosphorsäure) oben und die Kathode (Kathodenlösung mit Äthanolamin oder Diäthylamin und Saccharose) unten befand. Die Trennung fand bei $4^{\circ}$ und einer Spannung von 500 Volt über einen Zeitraum von $48-72$ Stdn. statt. Nach der Trennung, abzulesen an einer konstanten Einstellung der Stromstärke, wurde die Säule bei gleichbleibender Ausflußgeschwindigkeit (etwa $1 \mathrm{~m} / / \mathrm{Min}$.) in Fraktionen von $1 \mathrm{ml}$ entleert.

Die Extinktionsmessungen erfolgten in dem Spektrophotometer Typ PMQ 2 der Fa. Zeiss bei $280 \mathrm{~nm}$ und bei $470-480 \mathrm{~nm}$. Die pHWerte der Fraktionen bestimmten wir mit einer Einstabmeßkette der Fa. Ingold HA 404 Nr. A 7653 am pIH-Meter der Fa. Knick; Berlin.

Immunelektroploresen wurden nach der Methode von SCIIEIDEGGER (8) und Farnstoff-Stärkegelelektroplsoresen nach den Vorschriften von EDelman und Poulik (9) durchgeführt. Die Reinigung des Trans- 
ferrin-Eisen-Komplexes von nicht an Protcin gebundenem Eisen erfolgte durch Gelfiltration auf Sephadex G-200.

Bei den Versucber mit ${ }^{50} \mathrm{Fe}$ wurden pro Versuch, etwa $0,4 \mathrm{mC}$ verwendet. Dic Aktivität der Fraktionen bestimmten wir in dem Gamma-Probenwechsler der Fa. Packard mit einem 3"-Thalliumaktivierten Na-Jodid-Kristall. Eisen wurde nach der Bathophenanthrolinmethode nach PeTERs und Mitarbeitern (10) bestimmt. Für die Bestimmung wurde der Chemikaliensatz der Fa. Chemische Fabriken Asal, Schwalbach, verwendet.

\section{Ergebnisse}

\section{Isoelektrische Trennung von eisenfreiem Transferrin}

Eisenfreies Transferrin stellt sich in Ampholyten des pH-Bereiches 3-10 zwischen pH 5 und $\mathrm{pH} 6$ ein. Bei genauerer Untersuchung dieses $\mathrm{pH}$-Bereiches in Ampholyten $\mathrm{pH} 5-7$ wurden, wie aus Abbildung 1 hervor-

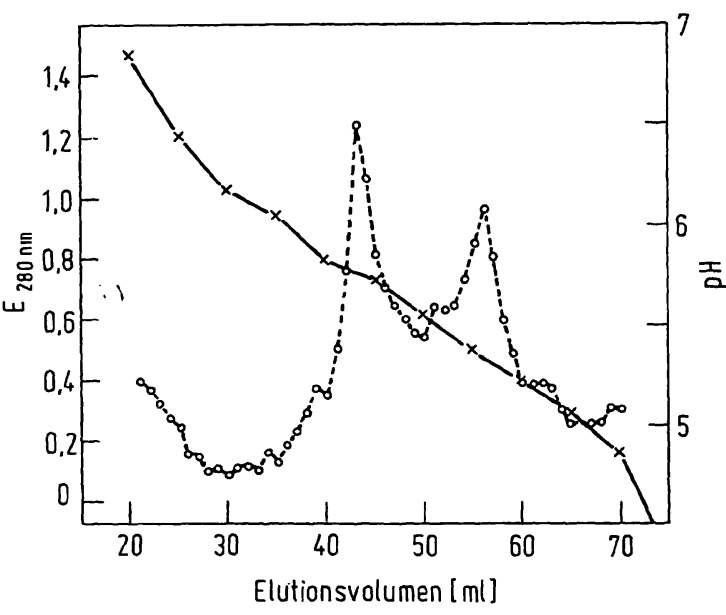

Abb. 1

Trennung von eisenfreiem Transferrin $(20 \mathrm{mg})$ in Ampholyten pH 5-7. Extinktion bei $280 \mathrm{~nm}(0--0)$ und pH-Werte $(x-x)$ der einzelnen

geht, deutlich 2 Komponenten getrennt, deren isoelektrischer Punkt bei pH 5,78 und pH 5,35 liegt. Dieses Ergebnis war gut reproduzierbar $(\mathrm{pH} 5,87, \mathrm{pH} 5,40)$. Immunelektrophoretisch entsprachen die genannten Extinktionsmaxima im Ansatz mit Antihumanserum nach Form und Lage des Präzipitates Transferrin. Zur Frage, ob sich die so gewonnenen Fraktionen elektrophoretisch trennen lassen, wurden die einzelnen Fraktionen nach Entfernung des Ampholyten durch Dialyse in der Harnstoff-Stärkegelelektrophorese untersucht. Wie Abbildung 2 zeigt, haben die beiden Fraktionen eine gleich große elektrophoretische Beweglichkeit, die auch mit derjenigen des Ausgangsmaterials übereinstimmt. Der Ampholyt ( $\mathrm{pH} 5-7$ ) ist stärkegelelektrophoretisch weit bis zur Anode ausgezogen.

\section{Isoelektrische Trennung des Transferrin-Eisen-Komplexes}

$\mathrm{FeCl}_{3}$ wurde mit eisenfreiem Transferrin zur Bildung eines Transferrin-Eisen-Komplexes zusammengegeben. Dem $\mathrm{FeCl}_{3}$ wurde.vor der Bindung an Transferrin ${ }^{59} \mathrm{Fe}$ als Chlorid zugesetzt. Die an das Protein gebundene Eisenmenge entsprach dem Eisenbindungsvermögen von $1,25 \mu \mathrm{g}$ Fe pro $\mathrm{mg}$ Transferrin bei $\mathrm{pH} \mathrm{7,4.} \mathrm{Die} \mathrm{in}$ der Eisenmenge pro Versuch ( $20 \mu \mathrm{g}$ Transferrin) enthaltene Aktivität betrug $0,4 \mu \mathrm{C}$.

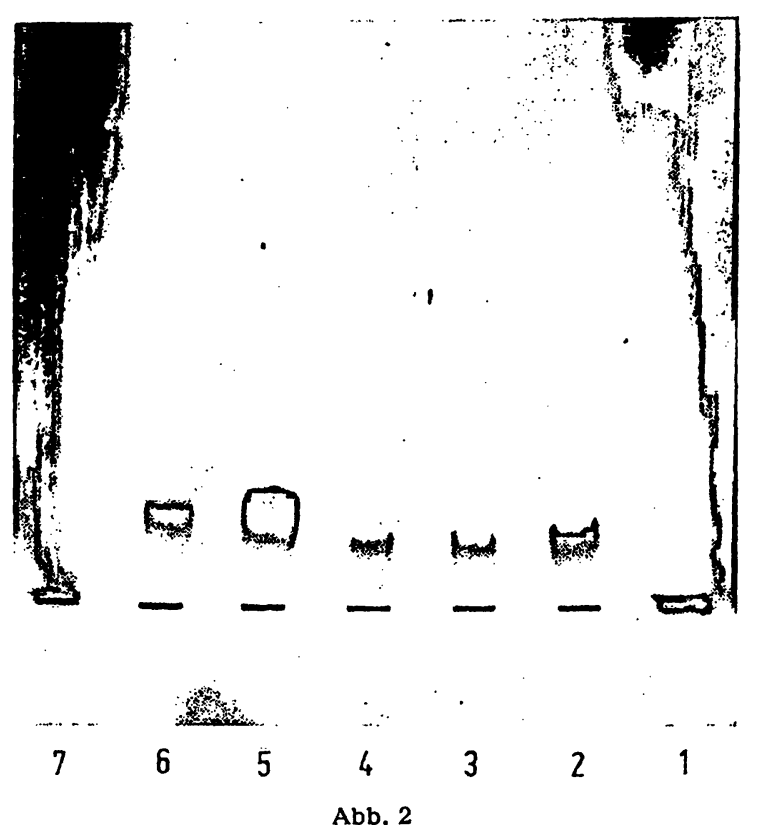

Harnstoff-Stärkegelelektrophorese des Ausgangsmaterials (4), der isoelektrisch getrennten eisenfreien Fraktion 1 (2) und 2 (3), der isoelektrisch getrennten eisenhaltigen Transferrin-Komponente 1 (5) und 2 (6) sowie des Ampholyten pH 5-7 (1 und 7)

Nach Eisenbelastung ergab die isoelektrische Trennung des Eisen-Transferrin-Komplexes in Ampholyten $\mathrm{pH}$ 3-10 eine Auftrennung in 2 Transferrin-Komponenten und zusätzlich in 1-2 Komponenten, die Eisen, jedoch kein Transferrin enthielten. Abbildung 3 zeigt die Proteinextinktion bei $280 \mathrm{~nm}$, die Extinktion des Transferrin-Eisenkomplexes bei $480 \mathrm{~nm}$, die Radioaktivität des Eisens und den Gesamteisengehalt. Es zeigte sich deutlich die Übereinstimmung sämtlicher Kurven bei beiden Protein enthaltenden Komponenten. Auffällig ist die Anreicherung von Eisen an der basischen Seite. Nach Gelchromatographie des Eisen-Transferrin-Komplexes an Sephadex G-200 zeigt die isoelektrische Trennung eine Abnahme des ersten, nur Eisen enthaltenden
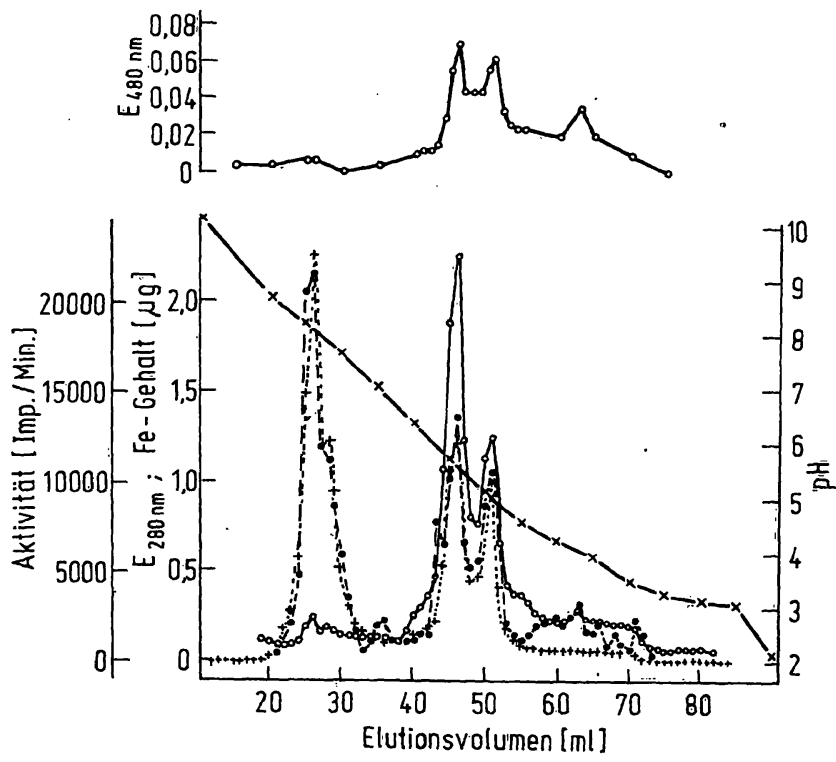

Abb. 3

Trennung von eisengesättigtem Transferrin in Ampholyten pH $3-10$. Oberes Diagramm: Verlauf der Extinktionskurve des Eisentransferrinkomplexes bei $480 \mathrm{~nm}$. Unteres Diagramm: Eisenaktivität in Imp./Min $(+--+)$, Extinktion bei $280 \mathrm{~nm}\left(0_{-}\right)$, Eisengehalt in $\mu \mathrm{g}\left(\cdot-0_{0}\right)$ und pH-Verlauf $(x-x)$ 


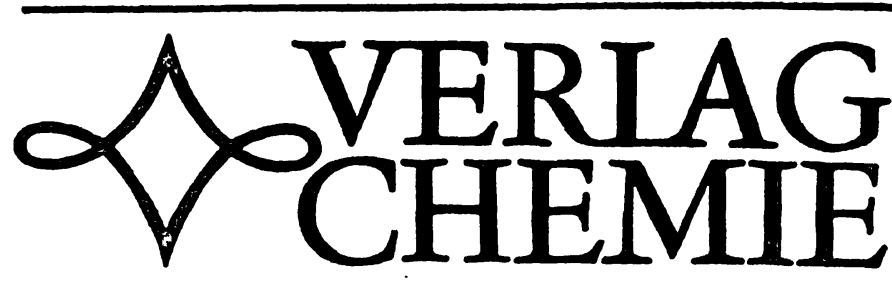

Soeben erscheint:

Knut Bäcbmann

\section{Messung radioaktiver Nuklide}

Kernchemie in Einzeldarstellungen. Band 2. Hrsgg. von Karl Heinrich Lieser. 1970. XII, 187 Seilen mit 194 Abbildungen und 30 Tabellen. Leinen DM 58, -

In den letzten Jahren hat sich das Gebiet der radioaktiven Meßtechnik stark weiterentwickelt. Konventionelle Detektoren wurden verbessert und vervollkommnet und neue Detektortypen geschaffen. In der Elektronik bedeutete die Transistorisierung cine wesentliche Erweiterung der Möglichkeiten. Aus der Notwendigkeit, gleichzeitig viele Proben zu messen, ergab sich die Vollautomatisierung und die Auswertung mit Computern.

Die Messung radioaktiver Strahlung ist heute nicht nur für die Physik wichtig, sondern sie spielt auch in der Chemie, Reaktortechnik, Biologie, Medizin und Geologie eine immer größere Rolle. Es erscheint deshalb angebracht, einen Uberblick über den gegenwärtigen Stand der radioaktiven Meßtechnik zu geben.

Das vorliegende Buch bietet eine zusammenfassende Darstellung aller Methoden der Messung radioaktiver Strahlung und wendet sich an Chemiker, Ingenieure, Biologen, Mediziner und Vertreter anderer naturwissenschaftlicher Fachrichtungen.

Dieses Werk gibt in erster Linie Anleitungen für die praktische Durchführung und Auswertung von Messungen. Die Vor- und Nachteile der einzelnen Meßanordnungen werden diskutiert, damit der Leser die für ihn optimalen Verfahren auswählen kann. Mit dem vorangestellten Kapitel über die Wechselwirkung von Strahlung mit Materie erleichtert das Verständnis der Wirkungsweise der Detektoren. Dem Aufbau der Detektoren und den elektronischen Hilfsmitteln sind aus dem gleichen Grund ebenfalls ein eigenes Kapitel gewidmet.

In der gleichen Reihe ist lieferbar:

Karl Heinrich Lieser

\section{Einführung in die Kernchemie}

Kernchemie in Einzeldarstellungen. Band 1. Hrsgg. von Karl Heinrich Lieser. 1969. XVI, 720 Seiten mit 230 Abbildungen, 103 Seiten Anhang, meist Tabellen. Lose beigefügte Nuklidkarte. Leinen DM 78,-

Das Buch bietet allen Naturwissenschaftlern eine gründliche Einführung in den gesamten Arbeitsbereich der Kernchemie. Das Gebiet ist in übersichtlicher, auch für den Anfänger verständlichen Form dargestellt.

Verlangen Sie unsere Sonderprospekte!

VERLAG CHEMIE - GMBH

WEINHEIM/BERGSTR.

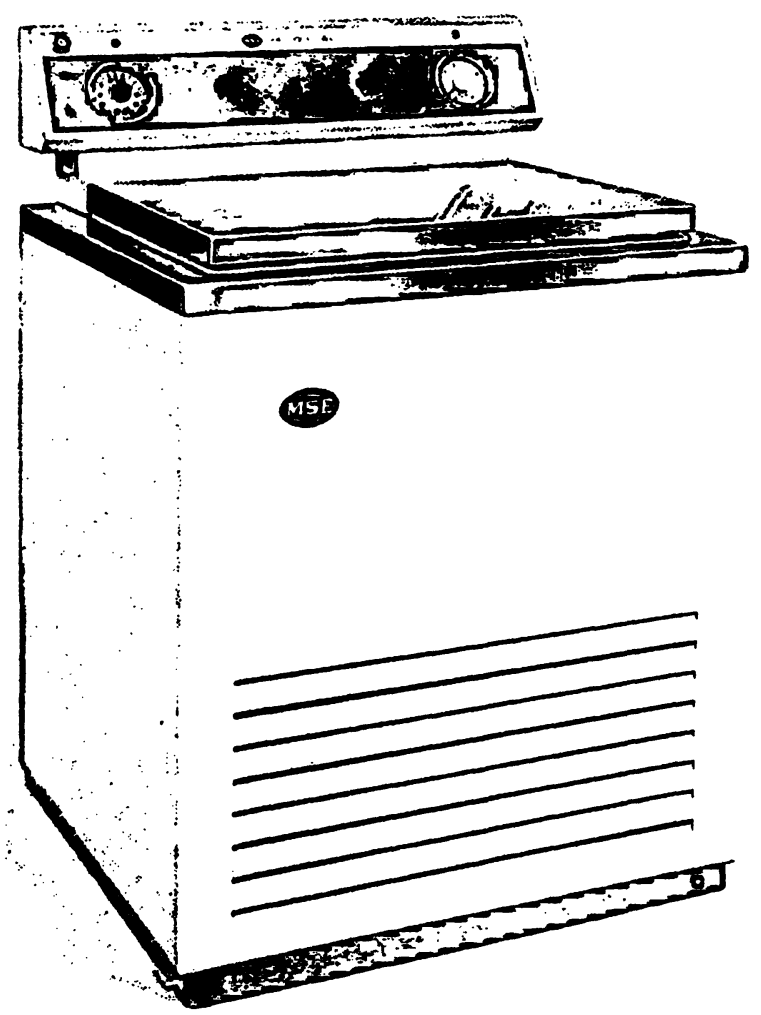

\section{MSE-Kühlzentrifuge Mistral 6 L}

Eine moderne Zentrifuge mit der außergewöhnlich hohen Kapazität von max. 6 Liter. Sie faßt z. B. sechs große Blutflaschen oder mit Vielfachträger bis zu 288 Reagenzgläser. Drehzahlen bis $6000 \mathrm{U} / \mathrm{min}$, mit » Superspeed « bis $22000 \mathrm{U} / \mathrm{min}$. Spezialrotor für Zonaltrennungen bei max. $5000 \mathrm{U} / \mathrm{min}$.

\section{Alleinvertretung für Deutschland: Colora Messtechnik GmbH 7073 Lorch/Württ., Postfach 5 T (07172) 6041, FS 07-248 886}

Technische Büros (Verkauf und Kundendienst): 1000 Berlin 30, Kurfürstenstraße 84, T 135200 2000 Hamburg 19, Osterstraße 63, T 4006 06, FS 02-12947 3000 Hannover, An der Tiefenriede 45, T 884500 4000 Düsseldorf, Kronprinzenstr. 62, T i 7860 , FS 08-587 253 6000 Frankfurt a.M.,Röderbergweg 4-6,T446031, FS 04-11216 8000 München 2, Dachauer Straße 175, T 5169858

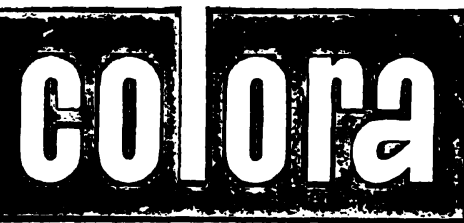




\section{GRADIPORE ELEKTROPHORESE}

Eine NEUE TECHNIK auf dem Gebiet der ELEKTROPHORESE!

(J. Margolis und K. G. Kenrick, Anal. Biochem. 25, Seite 347 [1968])

Diese Technik kombiniert Elektrophorese mit „MOLECULAR SIEVING“ durch die Elektrophorese auszuführen auf ein POLYACRYLAMIDE GRADIENT GEL: CONCAVE GRADIENT 4-26\% POLYACRYLAMIDE.

\section{ANWENDUNGSGEBIETE}

\section{EIWEISS-ANALYSE}

a) Trennung von Eiweißen nach Molekular-Gewicht und Struktur

b) Identifation von Eiweißen

c) Kontrolle von Homogenität

d) Geschätzte Festsetzung von Molekular-Gewicht durch Vergleichung mit bekannten Eiweißen

e) Festsetzung relative Eiweiß-Konzentrationen

\section{PREPARATIVE EIWEISS-FRAKTIONIERUNG}

III. Neue Forschungsmöglichkeiten für SEROLOGIE - IMMUNOLOGIE ENZYMPATHOLOGIE - HISTOLOGIE

ANFRAGEN: N.V.TEMPUS: IMPORTERS OF GRADIPORE:

OEGSTGEEST - Holland, Jan Steenlaan 21 oder Postfach 170 - LEIDEN - Holland

Sie wünschen sich ein Gerät, das die täglich anfallenden Arbeiten mit dem Mischen, Schütteln, Emulgieren, Herstellen von Suspensionen in Reagenzgläsern, Kolben, Flaschen etc. erleichtert. Wir haben den REAX gebaut. Sie verlangen, daß so ein Gerät rutschfest ist und nicht auf dem Labortisch umherwandert. Wir haben den REAX auf Saugfüße gestellt. Sie haben bei lhrer Arbeit nicht immer eine Hand frei, um auch noch Schalter zu bedienen. Wir haben einen Kontakt eingebaut, der den REAX bei Berühren mit dem Gefäß in Gang setzt. Und auch wieder abschaltet. Sie haben kaum noch Platz auf dem Labor-

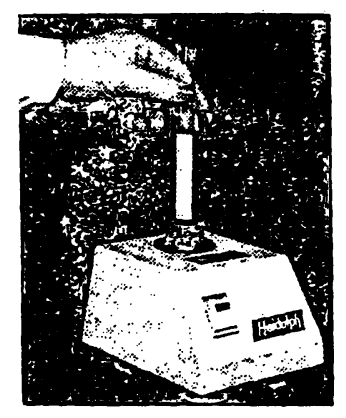
tisch. Wir haben den REAX

kleiner gebaut. Sie bestehen auf Wartungsfreiheit. Wir haben den REAX mit einem Spaltpol-Motor ausgerüstet, für den wir Dauerlauf garantieren. Sie könnten den REAX wirkungsvoll einsetzen? Wir haben dieses Gerät und auch noch andere.

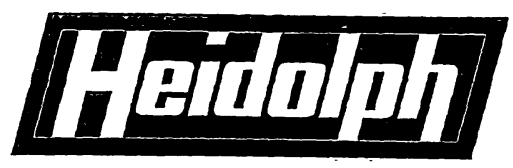

HEIDOLPH-ELEKTRO KG D-8420 KELHEIM
Soeben erscbien:

\section{Wasser}

Untersuchung - Beurteilung - Aufbereitung - Chemie Bakteriologie - Biologie

\section{Von Karl Höll}

Regierungsrat a. D., Dipl.-Lebensmittelchemiker, Hameln

Unter Mitarbeit von Helmut Peter und Dietrich Lüdemann

5., völlig neubearbeitete und erweiterte Auflage

Groß-Oktav. Mit 24 Abbildungen in 129 Einzeldarstellungen. XVI, 423 Seiten. 1970. Plastikeinband DM 52,-

Die 5. Auflage wurde dem neuesten Stand der Wissenschaft und Technik auf dem Gebiet "Wasser" angepaßt, die zahlreichen und mannigfaltigen Publikationen wurden bis Ende 1969. berücksichtigt. Das Buch ist nach wie vor in erster Linie dem Trinkwasser gewidmet. Eingehend behandelt werden auch die Gebiete Betriebs-, Bade-, Fluß- und Abwasser. Die Filtertechnik (z. B. Mehrschichtfiltration) wurde in Verbindung mit der Chemie des Wassers neu behandelt.

\section{Walter de Gruyter \& Co $\cdot$ Berlin}


Gradienten im Vergleich mit dem Transferrin-EisenKomplex (Abb. 4). Das Ergebnis der Trennung des Eisen-Transferrin-Komplexes in Ampholyten des $\mathrm{pH}$ Bereiches 5-7 ist im Vergleich mit den Befunden eisenfreien Transferrins in Tabelle 1 wiedergegeben. Wie deutlich aus den Werten hervorgeht, wird auch eisenhaltiges Transferrin in zwei Fraktionen getrennt, allerdings liegen die isoelektrischen Punkte dieser Komponenten um etwa $0,3 \mathrm{pH}$-Einheiten niedriger als diejenigen eisenfreien Transferrins.

Zur Klärung der Frage, ob die in Abbildung 3 dargestellten und nach Filtration an Sephadex G-200 abnehmenden eisenhaltigen, transferrinfreien Komponenten (Abb. 4) durch nicht an Protein gebundenes Eisen bedingt sind, wurde ein Ansatz mit Ampholyt ( $\mathrm{pH}$ 3-10) unter Zugabe von $15 \mu \mathrm{g} \mathrm{FeCl}_{3}+0,4 \mu \mathrm{C}{ }^{69} \mathrm{Fe}$ elektrofokussiert. Sofort nach der $\mathrm{FeCl}_{3}$-Zugabe zu der spezifisch leichteren Lösung, trat eine Braunverfärbung ein, die durch Hydrolyse des $\mathrm{FeCl}_{3} \mathrm{zu} F e(\mathrm{OH})_{3}$ bedingt sein dürfte. Die höchste ${ }^{59} \mathrm{Fe}-$ Aktivität lag in dem bereits aus anderen Versuchen bekannten pH-Bereich $\mathrm{pH}$ 8-9 (Abb. 5).

In der Stärkegelelektrophorese wies der TransferrinEisenkomplex eine größere Beweglichkeit auf als eisenfreies Transferrin.

\section{Diskussion}

Die vorliegenden Ergebnisse zeigen, daß eisenfreies Transferrin in Ampholyten des pH-Bereiches 3-10 und 5-7 in mindestens 2 Komponenten aufgetrennt werden kann, deren isoelektrische Punkte zwischen $\mathrm{pH} 5$ und $\mathrm{pH} 6$ liegen. Durch genaue Analyse in Ampholyten des $\mathrm{pH}-$ Bereiches 5-7 lassen sich die isoelektrischen Punkte der beiden Fraktionen mit pH 5,8 und pH 5,4 definieren. Immunelektrophoretisch handelte es sich bei beiden Komponenten um Transferrin. Allerdings kann aufgrund der Ergebnisse nicht ausgeschlossen werden, daß mit Ampholyten eines kleineren pH-Bereiches (z. B. $\mathrm{pH} 5-6)$ noch eine weitere Auftrennung zu erreichen ist.

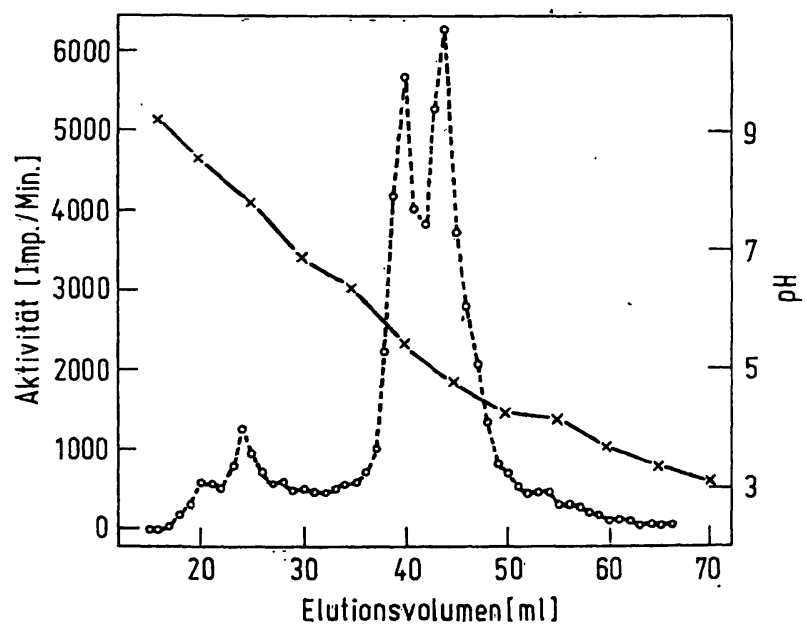

Abb. 4

Trennung von eisengesättigtem Transferrin in Ampholyten $\mathrm{pH}_{3-10}$ Entfernung des nicht an Protein gebundenen Eisens durch
filtration, Radioaktivităt $(0---0)$ und $\mathrm{pH}-\mathrm{W}$ erte $(x-x)$
Es stellt sich die Frage, ob die durch isoelektrische Trennung erhaltenen Fraktionen den genetischen Typen des Transferrins entsprechen. Diese werden nach ihrer Beweglichkeit in der Stärkegelelektrophorese als Transferrin $B, C$ und $D$ bezeichnet und weisen mit ihren zahlreichen Untergruppen eine geographische bzw. ethnographische Gebundenheit auf (11). Sowohl das Ausgangsmaterial wie auch die einzelnen Komponenten nach isoelektrischer Trennung wurden im Stärkegel elektrophoretisch untersucht. Nach 48 stdg. Dialyse zur Entfernung des Ampholyten stellten sich beide Fraktionen als Komponenten gleicher elektrophoretischer Beweglichkeit dar. Es ist deswegen unwahrscheinlich, daß sie verschiedenen genetischen Typen zuzuordnen sind, obwohl im Verlauf der Präparation aufgetretene und mit einer Denaturation verbundene Veränderungen nicht völlig auszuschließen sind.

Im Zusammenhang mit.der Heterogenität von Transferrin ist insbesondere ein unterschiedlicher Sialinsäuregehalt einzelner Komponenten zu diskutieren. Eine derartige Ursache wird - wie eingangs erwähnt - von JEPPSON und SJöQUIST (5) zur Erklärung des chromatographischen Verhaltens von Transferrin an Carboxymethylcellulose herangezogen. Die gleiche elektrophoretische Beweglichkeit der von diesem Autor beobachteten 2 Hauptkomponenten im Stärkegel bei unterschiedlichem Sialinsäuregehalt scheint eine Parallele zu den eigenen hier mitgeteilten Beobachtungen zu bilden. Die von Jeppson und SJöQuist beschriebene Unabhängigkeit der elektrophoretischen Beweglichkeit vom Sialinsäuregehalt steht im Widerspruch zu den Beobachtungen von PARKer und BEARN (12), die durch einen stufenweisen Abbau der 4 Sialinsäurereste des Transferrins durch Neuraminidase einen charakteristischen Wandel des elektrophoretischen Musters erzielten. Möglicherweise beruht der eingangs ( $1-4)$ erwähnte unterschiedliche Sialinsäuregehalt von Transferrin aus dem Serum, dem Liquor cerebrospinalis und dem Nabelvenenblut auf einem partiellen enzymatischen Abbau. In einer 1967 erschienenen Arbeit berichtet JEpPson (13)

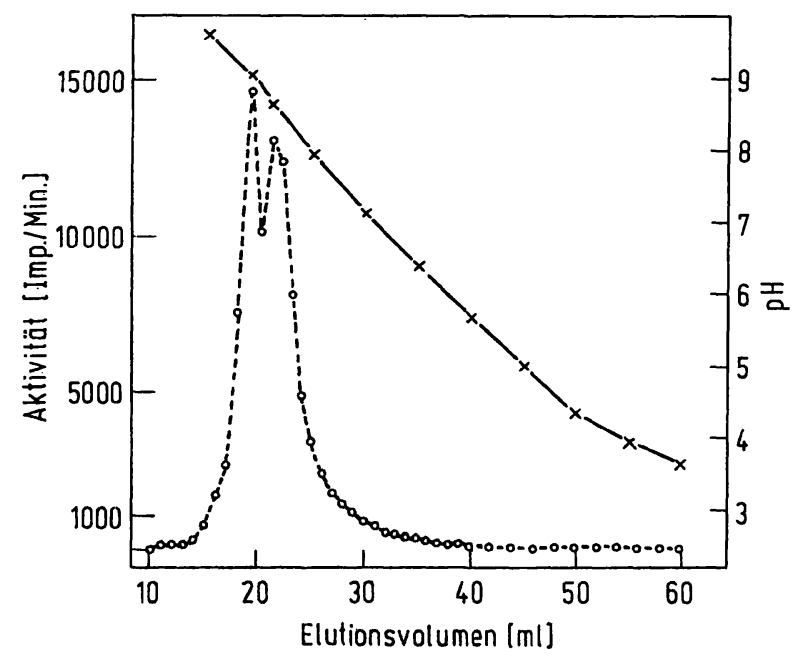

Abb. 5

Verlauf der Radivaktivität $(0--\infty)$ nach Trennung eines Ampholyt-(pH 3-10)-Eisengemisches mit Angabe der pH-Werte $(x-x)$ 
über die Trennung von Transferrin CD 1 in die beiden genetischen Typen. Gleichzeitig fand er nach tryptischer Hydrolyse in den Fingerprints der Peptide charakteristische Unterschiede für jedes individuelle Transferrin. Es scheinen demnach auch Unterschiede in der Primärstruktur innerhalb des Transferrinsystems zu existieren. $\mathrm{Ob}$ dies auch für die in unserem Fall beobachtete Heterogenität zutreffen könnte, muß offenbleiben.

Die Untersuchung des Eisentransferrin-Komplexes ließ ebenfalls eine Auftrennung in die 2 Fraktionen erkennen. In den Ergebnissen der isoelektrischen Fraktionierung von eisenhaltigem Transferrin zeigte sich schon im pH-Bereich 3-10 eine reproduzierbare Auftrennung in 2 Fraktionen. Die Analyse des Eisen-Transferrin-Komplexes in Ampholyt $\mathrm{pH}$ 5-7 ergibt isoelektrische Punkte bei $\mathrm{pH} 5,55(5,46)$ und $\mathrm{pH} 5,10(5,12)$. Die Ergebnisse zeigen eine gute Übereinstimmung mit den Befunden von WeNN und Wirliams (14), die nach isoelektrischer Trennung von ${ }^{59} \mathrm{Fe}$-markiertem Humanserum die Radioaktivität in zwei Gipfeln wiederfanden, die in Ampholyten des pH 5,5 und pH 5,2 lagen. $\mathrm{Da}$ die beiden isoelektrisch getrennten Komponenten des Eisen-Tranșferrin-Komplexes stärkegelelektrophoretisch schneller wanderten als die beiden eisenfreien Komponenten, untereinander (Eisen-Transferrin I und II und eisenfreies Transferrin I und II) jedoch keine Unterschiede in der elektrophoretischen Mobilität zeigten, können Konfigurationsveränderungen als Ursache der durch Eisenbindung an das Transferrin bedingten Veränderung angenommen werden. Wie aus den Ergeb-
Tab. 1

Ergebnisse isoelektrischer Messungen an eisenhaltigem und eisenfreiem Transferrin

\begin{tabular}{lcccc}
\hline & \multicolumn{2}{c}{ Eisenfreies } & \multicolumn{2}{c}{$\begin{array}{c}\text { Eisenhaltiges } \\
\text { Transferrin }\end{array}$} \\
& Versuch 1 & Versuch 2 & Versuch 1 & Versuch 2 \\
\hline $\begin{array}{l}\text { Isoelektrischer Punkt } \\
\text { Fraktion 1 }\end{array}$ & pH 5,87 & pH 5,78 & pH 5,55 & pH 5,46 \\
$\begin{array}{l}\text { Isoelektrischer Punkt } \\
\text { Fraktion 2 }\end{array}$ & pH 5,40 & pH 5,35 & pH 5,10 & pH 5,12 \\
\hline
\end{tabular}

nissen ersichtlich ist ( $\mathrm{Tab} .1$ ), beeinflußt die Bindung an Eisen die Ladungsverhältnisse im Hinblick auf den isoelektrischen Punkt deutlich. Dies entspricht den Befunden von KeLler und PENNel (15), die den isoelektrischen Punkt des eisengesättigten Transferrins bei $\mathrm{pH}$ 5,45 und denjenigen des eisenfreien Transferrins bei pH 5,8 fanden. Auch in der Harnstoff-Stärkegelelektrophorese wird die Wanderungsigeschwindigkeit des Transferrins durch Eisenbindung beschleunigt. Weiterhin fiel ein starker Gipfel, sowohl der ${ }^{59} \mathrm{Fe}$-Aktivität als auch des Gesamteisens auf, der bei $\mathrm{pH}$ 8-9 lag und keine Extinktion bei $280 \mathrm{~nm}$ und $480 \mathrm{~nm}$ zeigte. Der hohe Eisengehalt spricht für das Vorhandensein von Eisen, das durch den Ampholyten komplex gebunden ist. Das fast völlige Verschwinden dieser Komponente nach Gelfiltration zeigt, daß diese Bande durch nicht an Protein gebundenes Eisen verursacht ist. Der an dieser Stelle nach Gelfiltration noch angedeutet erkennbare Gipfel (Abb. 4) kann durch Freisetzung von Eisen aus dem Eisen-Transferrin-Komplex durch den Ampholyten zustande kommen.

\section{Literatur}

1. Williams, J., Biochem. J. 83, 355, (1965). - 2. Parker, W. C. und A. G. BeArN, J. exper. Med. 115, 83 (1962). - 3. HeIDE, K. und H. HAUPT, Behring-Werke Mittlg. 43, 161 (1964). - 4. Surron, H. E. und B. H. Bowman, Second Int. Confer. of Human Genetics, Rome 1961, Abstr. No. 327. - 5. Jeppson, J. O. und J. SJöQuist, Biochim. biophysica Acta (Amsterdam) 78, 658 (1963). - 6. Vesterberg, O. und H. Svensson, Acta Chem. Scand. 21, 206 (1967). - 7. ReIs, H. E. und O. WeTTER, Klin. Wschr. 47, 426 (1969). - 7a. ReIs, H. E. und O. WeTrER, Klin. Wschr. 48, 643 (1970). - 8. ScheIDEgGer, I. I., Internat. Arch.
Allergy 7, 103 (1955). - 9. Edelman, G. M. und M. O. Poulrk, J. exper. Med. 113, 861 (1961). - 10. Peters, T., T. J. GrovanNiello, L. Apta und J. F. Ross, J. Laborat. Clin. Med. S. Louis 48, 280 (1956). - 11. SEPpäLÄ, M., An. Med. Exper. biol. Fenniae, Suppl. 4, 43, 7 (1965). - 12. Parker, W. C. und A. G. Bearn, Science Washington 133, 1014 (1961). - 13. JeppsoN, J. O., Biochim. biophysica Acta (Amsterdam) 140, 468 (1967). - 14. WeNN, R. V. und J. Williams, Biochem. J. 108, 69 (1968). 15. Keller, W. und R. B. PenNEL, J. Laborat. Clin. Med. S. Louis 53,638 (1959).
Dr. H. E. Reis Dr. O. Wetter, P. D. Dr. R. Pfeiffer 43 Essen Hufelandstr. 55 